\title{
Optimization of plasma sampling depth and aerosol gas flow rates for single particle inductively coupled plasma mass spectrometry analysis
}

\author{
Ildikó Kálomista, Albert Kéri, Gábor Galbács* \\ Department of Inorganic and Analytical Chemistry, University of Szeged, Dóm square 7, 6720 Szeged, Hungary
}

\section{A R T I CLE IN F O}

\section{Keywords:}

SpICP-MS

Plasma sampling depth

Aerosol dilution gas flow

Carrier gas flow

Nanoparticles

\begin{abstract}
A B S T R A C T
We performed experiments to assess the separate and also the combined effect of the sampling depth and the aerosol gas flow rates on the signal formation in single particle inductively coupled plasma mass spectrometry (spICP-MS) measurements by using dispersions containing Ag and Au NPs. It was found that the NP signal can significantly be improved by the optimization of the sampling depth. With respect to the ,robust" setting, a signal improvement of nearly $100 \%$ could be achieved, which translates into a $25-30 \%$ improvement in size detection limits. It was also found that the shape of the spICP-MS signal histograms also change with the change of the plasma sampling depth. It was demonstrated that nanoparticle peak separation can also be significantly enhanced by using sampling depth optimization.

The effect of the aerosol dilution gas flow, now standard in most ICP-MS instruments, on the spICP-MS signal formation was also studied for the first time in the literature, as this flow was hoped to make spICP-MS measurements more practical and faster via the on-line dilution of the aerosol generated from nano-dispersions. Our experimental results revealed that the dilution gas flow can only be used for a moderate aerosol dilution in spICP-MS measurements, if the gas flow going to the pneumatic nebulizer is proportionally lowered at the same time. This however was found to cause a significant worsening in the operation of the sample introduction system, which gives rise to a strong NP signal loss. Thus it was concluded that the use of the aerosol dilution gas flow, in its present form, can not be suggested for spICP-MS analysis.
\end{abstract}

\section{Introduction}

Inductively coupled plasma mass spectrometry (ICP-MS) is a well established (ultra)trace analytical technique, routinely used for the elemental or isotopic analysis of liquid samples $[1,2]$. Its excellent detection limits and high selectivity are also often exploited for the localized or mapping analysis of various sectioned solid or tissue samples via the use of laser ablation sample introduction (LA-ICP-MS) [3,4], or for organometallic/speciation analysis where it serves as an element selective detector coupled to high performance liquid chromatography or gas chromatography $[5,6]$.

Single particle ICP-MS (spICP-MS) is a novel technique applicable to the analysis of nanoparticles, made possible by the attogram-range limits of detection of ICP-MS. This approach, which was introduced and first explored by Degueldre et al. in a series of papers around 2003, is now being found very useful in the literature for the rapid characterization of nano-dispersions [7-11]. The concept of spICP-MS is that since the atoms are aggregated in the nanoparticles (NPs), the NPs will generate spikes in the time-resolved signal, the intensity of which will be proportional to the mass of the analyte present in the particle. Through the statistical analysis of these spikes, information about the size distribution, number concentration and composition of NPs can be obtained $[8,9]$. The technique is nowadays considered to be a faster and more practical alternative to other established NP characterization methods such as SEM or DLS [12].

Due to its novelty, the effects of ICP-MS experimental parameters on nanoparticle measurements have been far less studied than for solution measurements. The most studied experimental parameters are the dwell-time (integration time), the particle concentration in the nano-dispersion and the acquisition time. It has been established that with routine quadrupole ICP-MS instruments, a few ms dwell-time and a particle concentration lower than approx. $10^{5} \mathrm{~mL}^{-1}$ is optimal for the spICP-MS measurements. Under these conditions, as little as $100 \mathrm{~s}$ acquisition time accumulates signals from over ten thousand particles, which results in good quality signal histograms and reliable results $[9,13]$. The effect of the use of the collision cell and spectral interferences have also been investigated in a couple of studies [13,14].

For solution ICP-MS, it is known that the plasma sampling depth, forward RF power and aerosol carrier gas flow rate should be jointly optimized for every element $[1,15,16]$. One of the most important optimization parameter is sampling depth (SD), which is the distance between the tip of the sampling

\footnotetext{
* Corresponding author.

Email address: galbx@chem.u-szeged.hu (G. Galbács)
} 
cone and the load coil. The optimum sampling depth depends on a number of factors including the ionization energy of the analyte, the analyte diffusion rate, the mean diameter of the aerosol droplets, the aerosol carrier gas flow rate, the sample matrix, the plasma RF power, etc. $[1,15-20]$. A higher RF power increases the plasma temperature, a lower carrier gas flow rate and smaller droplet size decreases the neccessary residence time of the droplet in the plasma, which increases the ionization degree and this decreases the sampling depth at which the signal is maximal. However at a too low SD value, the ionization degree will be lower, which gives rise to a lower signal. Simulation results also indicate that increasing the sampling depth will slightly also decrease the plasma temperature at the orifice of the sampler, which again makes the signal lower [21]. The volume of the analyte ion cloud will increase while progressing in the plasma central channel, and the lower is the analyte mass the higher is the rate of diffusion. In order to efficiently sample the ion cloud, it still has to be compact, thus the optimal SD will be shorter; thus for lower mass, lower ionization energy analytes the SD optimum tends to be lower [17]. Recently, Ho et al. [22] pointed out to that the optimal sampling depth in solution-mode ICP-MS depends more on the boiling point of the element or the corresponding oxide (the latter being relevant because the dried aerosol from most metallic analytes present in a nitric acid solution will be oxides) rather than the ionization potential of the element. The authors experimentally found a reasonable, positive correlation between the optimum sampling depth values and the boiling point of both the elements and their oxides, whereas the ionization potential was found to have little influence.

Although signal optimization is routine in solution ICP-MS practice, but the objective function - provided by the ,tuning protocols" pre-programmed in the instrument software - is usually a composite one, as it tries to provide ,robust” operating conditions (e.g. maximize the analyte signal for pre-set isotopes, minimize signal scatter, minimize the $\mathrm{CeO} /$ Ce signal ratio, minimize the maintenance of interface components, etc. all at the same time). The result is that a compromise value, usually in the $8-10 \mathrm{~mm}$ range, is generally suggested to be used $[15,16,18,23]$.

The optimization of the instrumental parameters with the objective of obtaining the largest ion signal is obviously would also be very important in spICP-MS analysis, as this could lower the NP size detection limit. This would be especially important, since the attention of nanoscience is more and more turning towards the sub-10 nm size range [24-26]. However, tuning protocols have not yet been worked out for the measurement of nanoparticles. Generally the ICP-MS is set up according to solution-based principles and robust plasma and data acquisition conditions are used also for spICP-MS experiments [9,11,27]. These typical settings include 8-10 mm sampling depth, $>1200 \mathrm{~W}$ RF power (facilitates the efficient ionization of the particles), and approx. $1 \mathrm{~L} / \mathrm{min}$ aerosol flow rate (compatible with most standard concentric nebulizers). The particle size calibration curves achieved with these settings run from approx. $20-200 \mathrm{~nm}$, which is equivalent to a 2-3 orders of magnitude range in terms of particle mass. The high end of this dynamic range is limited by the incomplete vaporization/ionization of the particles as well as that the short signal spikes originating from large mass nanoparticles can easily reach the trip point of the detector in pulse counting mode $[28,29]$. The only experimental data available in the literature on the optimization of the sampling depth in spICP-MS was very recently published by Ho et al. for Au and zirconia NPs [22].

As is known, ICP-MS instruments do not tolerate the direct nebulization of concentrated sample matrices. High total dissolved solid (TDS) matrices are generally recognised to cause a drift/drop of sensitivity due to e.g. the build-up of matrix deposits in the nebulizer or on the cones, an increase in viscosity, density, or surface tension that strongly affect aerosol formation, suppression of analyte ionization in the presence of high concentrations of easily ionizable elementsx, etc. Hence the widely accepted TDS limit is about $0.2 \%(2 \mathrm{~g} / \mathrm{L})$ for conventional ICP-MS instruments, also defined in standard methods such as EN-ISO 17294-2, US-EPA 6020B or EN 13,805 used in the analysis of environmental matrices [2]. To avoid these problems, modern ICP-MS instruments include a gas flow option that is meant to facilitate the measurement of high
TDS samples. This option is called „High Matrix Introduction” (HMI) in Agilent [23], ,Argon Gas Dilution” (AGD) in Thermo Scientific [30], and „All Matrix Solution” (AMS) in Perkin-Elmer [31] instruments. Essentially these approaches all simply employ an extra, controlled argon gas flow added to the sample aerosol stream between the spray chamber and the base of the torch in order to programmatically dilute the sample aerosol flow. In our view, the use of the aerosol dilution option may also have relevance in SpICP-MS analysis, because if the dilution can be consistently done in a reasonably wide concentration range, it could make the measurement of nanodispersions of unknown concentration faster and more practical.

The carrier gas flow in ICP-MS instruments usually also serves as the nebulizer gas flow for pneumatic nebulizers, thus the addition of the aerosol dilution gas (it will be abbreviated as HMI from this point on) flow to this increases the total argon gas flow to the plasma. It is well known in solution-mode ICP-MS that an increase in the carrier gas flow rate significantly modifies plasma conditions $[19,28]$, therefore the Agilent HMI matrix dilution software function also drastically decreases the gas flow rate entering the nebulizer thereby keeping the total flow close to the normal value. Although this approach will have a strong impact on the operation of the pneumatic nebulizer (a lower gas pressure will increase the size of the aerosol droplets and will also affect the efficiency of aerosol generation [32,33]), but in the case of quantitative solution measurements relying on long integration times (seconds range) and matrix-matched calibration, this is a working approach. At the same time, particle-mode, time-resolved ICP-MS measurements rely on the assumption that $i$ ) the nanodispersion is so much diluted that each aerosol droplet contains a maximum of one NP only, and ii) the aerosol itself is so dilute (the droplet number concentration is so low) that only one droplet is entering the plasma within each integration time window. This is neccessary to avoid time-resolved signal peak overlaps. Under these conditions, a change in the aerosol droplet size distribution or nebulizer efficiency may distort spICP-MS signals. Thus, the effects of the aerosol dilution and carrier (nebulizer) gas flows need to be studied in detail.

Considering the above, the goal of the present work was to perform a detailed experimental study of the effect of plasma sampling depth and argon gas flow rates related to sample introduction in an Agilent 7700x ICP-MS spectrometer on the performance of single particle measurements, with special regard to sensitivity. Commercial gold and silver nanodispersions were used as test samples, as these two elements are not only relevant for applications, but also because they represent analytes with significantly different masses, ionization energies and boiling points.

\section{Experimentals}

\subsection{ICP-MS instrumentation}

A quadrupole Agilent 7700X inductively coupled plasma mass spectrometer (ICP-MS), equipped with standard Agilent accessories, was used in all experiments. The sample introduction system consisted of an Agilent I-AS type autosampler, a MicroMist microflow concentric pneumatic nebulizer and a Peltier-cooled, Scott-type spray chamber. Data acquisition was performed in the time resolved analysis (TRA) mode, using settings typical for spICP-MS measurements (Table 1.). Please note that the Agilent MassHunter software uses a terminology different from other software (dwell time is called integration time), but for the sake of clarity, we use the conventional dwell time terminology here.

\subsection{Materials and methods}

A series of standard PELCO NanoXact nano-dispersions containing tannic acid capped gold and silver NPs and citrate stabilized silver NPs obtained from Ted Pella (Redding, California, USA) were used as samples. Gold and silver NPs were selected for the study to represent heavy and medium mass analytes, also with different ionization energies. The certified nominal size of the gold NPs used were 
Table 1

Overview of the spICP-MS acquisition parameters used.

\begin{tabular}{llll}
\hline Plasma parameters & Data acquisition parameters \\
\hline RF power: & $1550 \mathrm{~W}$ & $\begin{array}{l}\text { Measurement } \\
\text { mode: }\end{array}$ & $\begin{array}{l}\text { time } \\
\text { resolved } \\
\text { analysis } \\
6 \mathrm{~ms}\end{array}$ \\
$\begin{array}{l}\text { Argon gas } \\
\text { flow rate: }\end{array}$ & $20.0 \mathrm{~L} / \mathrm{min}$ & Dwell time: & $100 \mathrm{~s}$ \\
$\begin{array}{l}\text { Carrier gas } \\
\text { flow rate: }\end{array}$ & $\begin{array}{l}\text { 0.55-1.05 L/ } \\
\text { min }\end{array}$ & $\begin{array}{l}\text { Total } \\
\text { acquisition } \\
\text { time: } \\
\text { Measured } \\
\text { isotopes: }\end{array}$ & ${ }^{197} \mathrm{Au}$, \\
$\begin{array}{l}\text { Dilution gas } \\
\text { r.MI) flow }\end{array}$ & $0-0.5 \mathrm{~L} / \mathrm{min}$ & $\mathrm{Ag}$ \\
$\begin{array}{l}\text { Sampling } \\
\text { depth: }\end{array}$ & $3-15 \mathrm{~mm}$ & & \\
\hline
\end{tabular}

silver NPs were 30,60, $100 \mathrm{~nm}$. Nano-dispersions with the neccessary number concentrations were always prepared prior to the experiments on the same day, by using dilution of the stock colloids with trace-quality de-ionized labwater (MilliPore Elix 5 with Synergy, Merck, Darmstadt, Germany). Before dilution and also directly before aspiration into the ICP-MS, the nano-dispersions were sonicated for a short ime in an ultrasonic bath (Bransonic 300, Ney, Danbury, Connecticut, USA) in order to minimize particle aggregation. The $99.996 \%$ purity argon gas used was obtained from Messer (Hungary).

All data processing was performed within the Agilent MassHunter (Santa Clara, California, USA) and OriginLab Origin (Northampton, Massachusetts, USA) software. Data evaluation in spICP-MS uses the histograms (frequency-count diagrams) produced from the time-resolved signal traces. In histograms from correctly set-up experiments, the background signal shows up as a peak at a low count position, fittable by a symmetric, Poisson function. The histograms can be directly used for qualitative purposes, but it is useful to correct for the background. Here we adopted the approach that the mode of the background peak is subtracted from the mode of the particle peak (fittable by a lognormal function) [14]. All histogram operations were carried out using unit bin sizes, with the horizontal scale plotted in the 0-2000 range. The number of detection events was calculated as the integral of the particle peak in the histogram.

\section{Results and discussion}

\subsection{Optimization of sampling depth}

\subsubsection{NP signals and number of detection events}

Characteristic pulse intensities (modes of NP peaks in the histogram) were recorded in the $\mathrm{SD}=3-15 \mathrm{~mm}$ range, as is shown in Fig. 1 . The plasma RF power and carrier gas flow rate settings were left at their conventional values

a)

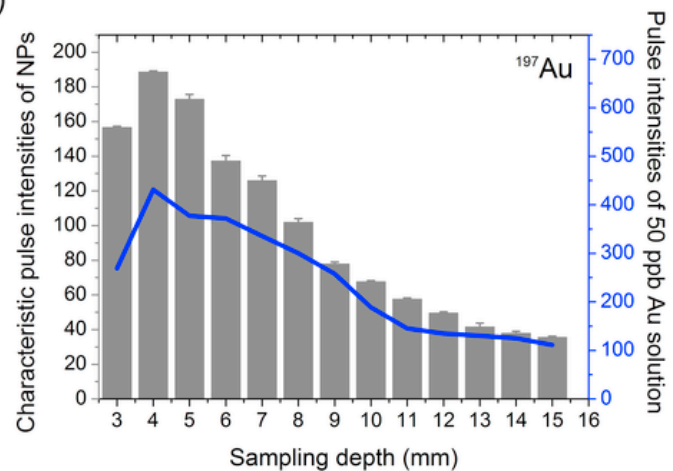

(Table 1.) during this experiment. As it can be observed in Fig. 1., the signal maximum is clearly at around $\mathrm{SD}=4 \mathrm{~mm}$ for both Au and Ag NPs. This is a significantly lower value than the robust values generally used $[9,11,27]$, but at the same time, the signal stability and repeatability clearly does not worsen with the decrease of the SD, but stays below $1-2 \%$ RSD (the error bars are barely visible in Fig. 1.), thus the optimum sensitivity is at around $4 \mathrm{~mm}$ for these two analytes. For comparative purposes, we also investigated the signal behaviour of $\mathrm{Ag}$ and $\mathrm{Au}$ solutions (measured in solution mode) with respect to the variation of sampling depth and found the same (approx. $4 \mathrm{~mm}$ ) optimum values, as is also shown in Fig. 1.

Data in Fig. 1. reveal very small differences in the behaviour of $\mathrm{Ag}$ and Au NP signals. The data for silver shows a somewhat faster decay of the particle signal (the signal at $15 \mathrm{~mm}$ drops to $14.7 \%$ of the maximum for $\mathrm{Ag}$, whereas it is $20.5 \%$ for $\mathrm{Au}$ ). This small difference in the signal behaviour probably can be explained by the slightly higher rate of diffusion of $\mathrm{Ag}$, due to its lower ionization energy and lower mass $(7.57 \mathrm{eV}$ for ${ }^{107} \mathrm{Ag}$ as opposed to $9.22 \mathrm{eV}{ }^{197} \mathrm{Au}$ ).

Earlier laser ablation and monodisperse dried aerosol ICP-MS $[17,34,35]$ studies showed that the time neccessary for the complete vaporization and ionization of nanoparticles is significantly longer than for dried aerosols originating from solutions. This would suggest that higher sampling depth values should be optimal for spICP-MS than for solutions. Nevertheless our results Fig. 1. for $\mathrm{Au}$ and $\mathrm{Ag}$, in accordance with the data presented by Ho et al. for Au and zirconia NPs [36] indicate highly similar (within one mm) optimum SD values for solution-mode and particle-mode measurements, at least for relatively small nanoparticles (up to $80 \mathrm{~nm}$ diameter), in spite of the quite different ionization potential and boiling point of these analytes. This suggests, that solution-mode ICP-MS sampling depth optimization curves can in fact potentially be used as guides during spICP-MS sensitivity (e.g. size detection limit) optimization. At the same time, a short sampling depth, which is optimal from the point of view of sensitivity will lower the upper limit of size calibration, as the vaporization/ionization of larger (e.g. over $100 \mathrm{~nm}$ ) nanoparticles need longer time, thus larger sampling depth values, as was shown by Ho et al. by simulation [36].

It is interesting that the shape of the NP signal histograms also changes with the change of the sampling depth (Fig. 2). By comparing histogram peak shapes it can be stated that NP histogram peaks get narrower and taller with an increase in SD, but the characteristic pulse intensity gets lower (as was alluded to earlier). This suggests that at low $\mathrm{SD}$ values the analyte cloud is more compact thus its sampling is more efficient, when neither the diffusion nor the recombination is significant. The larger width of the histogram peaks at low SD values probably indicates that the degree of ionization is less uniform within the analyte cloud.

At the same time the number of NP detection events, which can be calculated from the integral of the histogram curves, was found to stay fairly constant as a function of SD, with $1-5 \%$ relative standard deviations. This indicates that basically all NPs which are above the size detection limit are in fact detected independently from the sampling depth, at least in the range of

b)

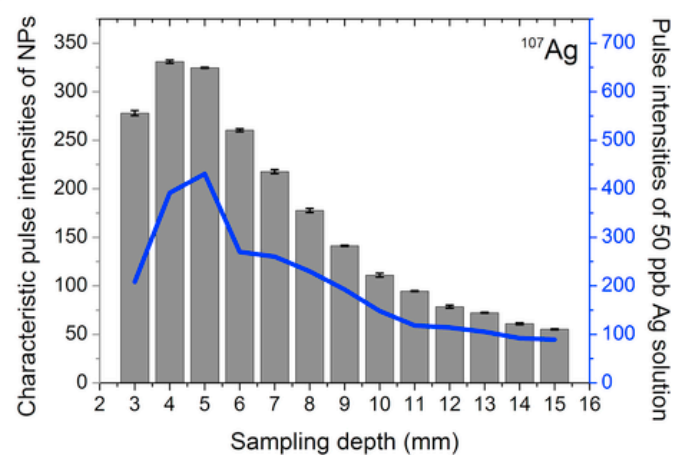


a)

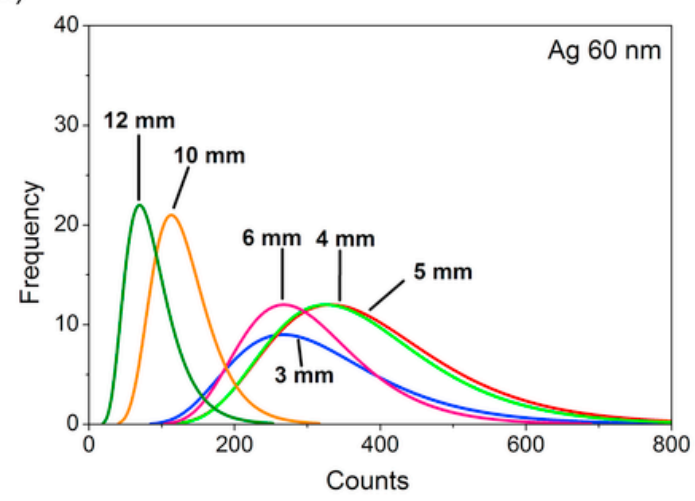

b)

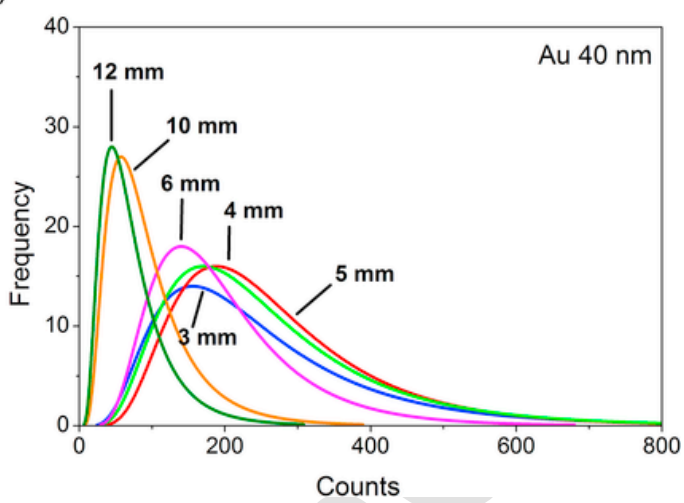

Fig. 2. The shapes of NP signal histograms as a function of sampling depth for Ag (panel A) and Au (panel B) nano-dispersions.

3-15 mm. In the case of the smaller Au NPs, there is only a slight, few percent (from 1580 to 1490) trendlike decrease in the number of detection events in this SD range. This is probably due to the lower signal and hence poorer $\mathrm{LOD}_{\text {size }}$ at higher SD settings, which results in that some smaller particles are not detected.

\subsubsection{Particle size detection limits}

The pre-requisite of the successful use of the spICP-MS technique is that the size (diameter) of the NPs in the nano-dispersion is significantly greater than the size detection limit for the given analyte isotope (it is generally assumed that the NPs have a spherical shape). As nanotechnology is starting to focus on the synthesis and investigation of smaller (1-10 nm) NPs, it is crucial that the size detection limit of spICP-MS, which is currently between 15 and $60 \mathrm{~nm}$ (mainly depending on the detection efficiency of the instrument as well as the ionization energy and relative abundance of the analyte isotope) $[8,13,37]$, should be significantly improved. The findings described in the preceeding section suggest that a contribution to this improvement is possible via the optimization of the sampling depth.

In the case of Au NPs, we found that the $\mathrm{LOD}_{\text {size, }}$ calculated as the diameter equivalent of three times the standard deviation of the blank signal in the size calibration plot $[8,14,38]$ improved from $19 \mathrm{~nm}$ to $13 \mathrm{~nm}$ when the sampling depth was decreased from $10 \mathrm{~mm}$ to $4 \mathrm{~mm}$. This is a relative improvement of $32 \%$. At the same time, in the case of $\mathrm{Ag}$ NPs, the $\mathrm{LOD}_{\text {size }}$ improved from $35 \mathrm{~nm}$ to $26 \mathrm{~nm}$ (a relative improvement of 26\%). This improvement is also illustrated by the two signal histograms in Suppl. Fig. 1. It can be observed that at $\mathrm{SD}=4 \mathrm{~mm}$ the peak from $30 \mathrm{~nm} \mathrm{Ag} \mathrm{NPs} \mathrm{can} \mathrm{be} \mathrm{clearly} \mathrm{separated} \mathrm{from} \mathrm{the} \mathrm{background}$ peak, whereas the two peaks coalesce at $\mathrm{SD}=10 \mathrm{~mm}$.

\subsubsection{Size resolution}

In most studies spICP-MS is used to find the characteristic (mean) particle diameter for NPs in a nano-dispersion, but in principle it is also capable of providing size distribution info on polymodal dispersions, as it has been demonstrated in a few studies [28,38-40]. For the success of such a measurement, the „NP size resolution” needs to be high enough to resolve the NP peaks. In Fig. 2. we showed that the optimization of the NP signal via decreasing the sampling depth largely widens the signal histograms. This can worsen the size resolution of the measurement, thus we also assessed the effect of SD on this performance parameter.

In order to characterize the NP size resolution, the classical, simple mass spectroscopy definition of resolution $(R=M / \Delta M)$, which was defined for symmetrical peaks, [41] can not be directly used as spICP-MS histogram peaks have an asymmetric, lognormal shape [42]. Therefore, we here chose to characterize the size resolution by calculating the net horizontal distance of two neighboring, deconvoluted NP peaks taken at their half heights (peak separation distance):

$$
d=M_{H I}-M_{L O}-R P W H M_{L O}-L P W H M_{H I}
$$

where $M_{H I}$ and $M_{L O}$ are the modes (characteristic intensities) of the particle peaks, whereas $L P W H M_{H I}$ and $R P W H M_{L O}$ are the left peak-width-at-half-maximum and right peak-width-at-half-maximum values for the larger and smaller NP peaks. The latter two values are introduced to take into account the asymmetry of the peaks; e.g. $R P W H M_{L O}$ is meant as the part of the peak width at the half maximum falling on the right hand side of the peak. Using this definition, the size resolution is higher, if the value of $d$ is larger for a given pair of NP peaks. Please note that this parameter was only introduced here to characterize the trend of changes in size resolution as a function of the sampling depth, and not to provide a general definition of size resolution. Its use is also based on the assumption that the two peaks are actually resolved, that is $d$ is positive.

By using a mixed dispersion of 60 and $100 \mathrm{~nm}$ NPs and the calculation described above, we found the size resolution monotonously increasing with the decrease of the sampling depth (Fig. 3.). This clearly indicates that the use of a lower, e.g. $4 \mathrm{~mm}$, sampling depth setting is beneficial not only from the point of view of the size detection limit, but also for the size resolution. The effect is similar for $\mathrm{Au}$ and $\mathrm{Ag}$, which suggests that it is element-independent.

\subsection{The effect of aerosol dilution gas flow}

We investigated not only the combined, but also the separate influence of HMI and CG gas flows on the nanoparticle signals and on the number of detection events, also in relation to the sampling depth. The aerosol dilution factor (DF) was defined as

$$
D F=\frac{C G+H M I}{C G}
$$

wherein CG and HMI denote the corresponding gas flow rates (in $\mathrm{L} / \mathrm{min}$ units). While varying the DF in the range of 1.0-1.9, the sum of CG and HMI flow rates was always kept constant at $1.05 \mathrm{~L} / \mathrm{min}$, the manufacturer suggested value for the MicroMist nebulizer. The dilution factor thereby expresses the relative increase in the total gas flow rate entering the plasma. We executed these experiments only with gold NPs because a) the sample introduction effects studied here should be independent from the quality of the NPs in the highly diluted nanodispersion, and b) sampling depth optimization described in the preceeding section revealed no significant difference in the behaviour of Au and Ag NPs.

\subsubsection{NP signals}

A mixed NP signal behaviour can be observed when the aerosol dilution is used (Fig. 4., panel A). At $\mathrm{SD}=5$ and $10 \mathrm{~mm}$, the characteristic signal does not significantly change if DF is increased. However, the situation is completely different at the two other sampling depth settings: with the increase of DF, the NP signal increases at $3 \mathrm{~mm}$, and decreases at $4 \mathrm{~mm}$. This suggests that with an increase of aerosol dilution (total gas flow), the signal optimum shifts to a lower sampling depth. 
a)

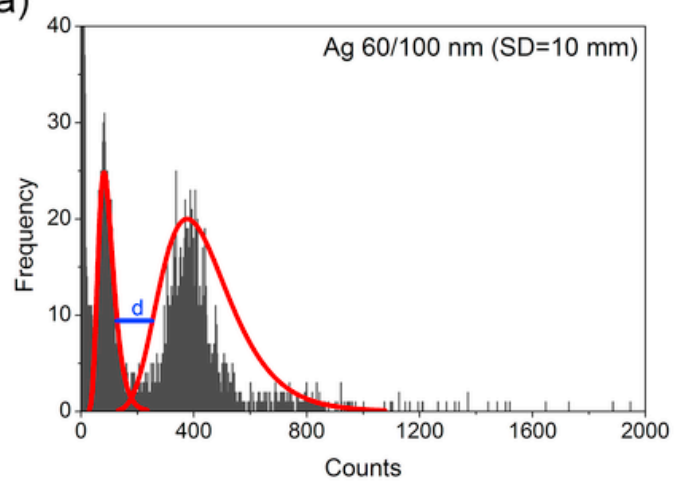

b)

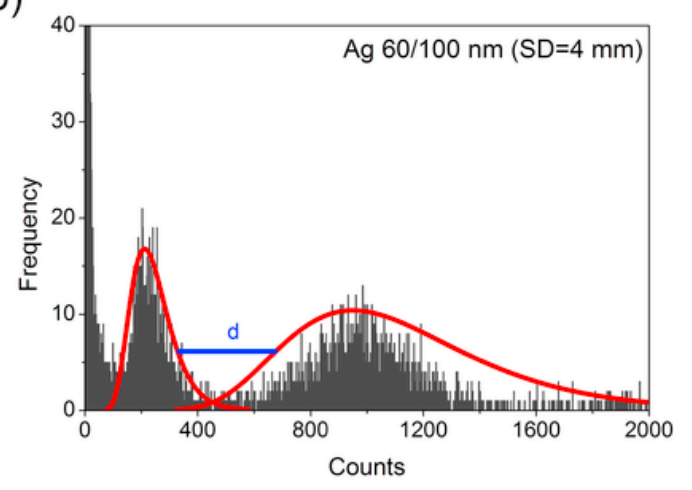

c)

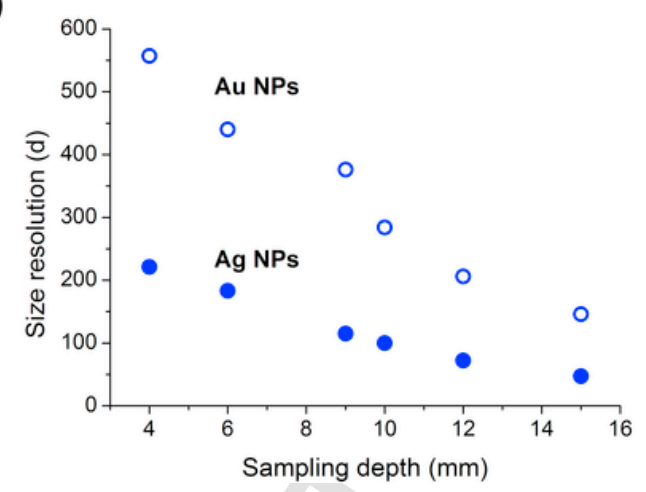

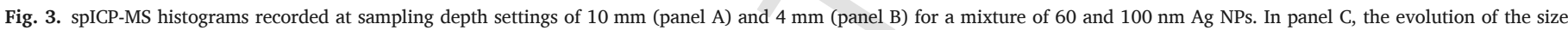
resolution ( $d$ parameter) is shown as a function of sampling depth for $\mathrm{Ag}$ and $\mathrm{Au}$, based on data collected by the measurement of a mixture of 60 and $100 \mathrm{~nm} \mathrm{NPs}$ for each.

The complexity of the processes can be assessed by also considering the observed individual effects of the change of HMI and CG gas flow rates on the signal, shown in panels B and C of Fig. 4. Please note that these two graphs depict situations where the total gas flow to the plasma was changing, unlike in panel A. As can be seen, if aerosol dilution is not used then decreasing the CG flow rate (from its normal $1.05 \mathrm{~L} / \mathrm{min}$ value) strongly decreases the signal, partly because of the lower nebulization efficiency. Apparently, this is not offset by the lower plasma cooling effect caused by the combiner effect of lower argon gas flow rate and lower mass transport rate into the ICP plasma. However, if CG is fixed at its normal value and an increasing rate of HMI flow is added to it, then the NP signal also strongly decreases after a moderate increase at first. Here, probably the increased plasma cooling effect and a shorter effective residence time provided by the increased total argon gas flow to the plasma is responsible for the signal loss. The overall pattern is that any deviation from the CG value optimal for the pneumatic nebulizer used causes a significant NP signal loss, even if the sum of the CG and HMI flow rates is kept constant.

The signal loss can also be explained by the change of flow conditions due to the geometry of the HMI injector glass piece. This glass piece is a simple, ball-jointed T-connector in Agilent 7700 instruments, which is inserted between the torch base and the exit port of the spray chamber. The HMI flow arrives to the piece from the side, thus the direction of the dilution gas flow is perpendicular to the aerosol flow. Obviously, this is an aerodynamically inadvantageous arrangement, which probably disturbs the flow of aerosol droplets. For optimum ICP-MS signal, the direction and rate of the aerosol gas flow is also very important, as it has been recently shown by Aghaei and Bogaerts in their solution droplet-evaporation simulation studies. It was found that the exact uniaxial positioning of the aerosol flow within the injector tube (on-axis) with the sampling orifice is crucial for an efficient analyte sampling through the sampling orifice [43]. Experiments and simulations carried out by Spencer et al. [44,45] also conclusively showed that any deviation of the analyte flow from the central axis has a negative effect on the transmission between the first and second vacuum stages. The Bogaerts group recently even showed [46], that backward and rotational flows can also occur in the ICP torch at increased aerosol carrier gas flow rates. It therefore seems reasonable to assume that the HMI flow in its present, factory arrangement disturbs the aerosol flow and pushes it to the side of the injector tube, thus forcing most of the particles to follow an off-axis flow path leading through a cooler, outer part of the plasma which also largely increases analyte loss at the sampling orifice.

\subsubsection{Number of detection events}

We also tested if the number of detection events occurring during a fixed acquisition time changes proportionally with the HMI gas flow rate, which would be a direct indication that aerosol dilution is in fact achieved. This may be beneficial during spICP-MS measurements. The number of detection events were calculated as the integral of the histogram peaks. The results can be seen in Fig. 5. in an arrangement similar to the one shown in Fig. 4.

Overall, the aerosol dilution generated by the HMI flow seems to be effective if the sum of the CG and HMI flow rates are kept constant: the decrease of the number of detected nanoparticles is approximately proportional with the dilution factor calculated (please note that the DF scale in panel A of Fig. 5. is not linear). This dilution effect also seems to be largely independent from the sampling depth. However, the content of panels B and C indicate that this ,aerosol dilution” is not directly affected by the HMI flow only. The increase of the HMI flow alone (in the same range of $0.0-0.5 \mathrm{~L} / \mathrm{min}$, as was used in the top panel to produce a DF range of 1.0-1.9) produces only a slight decrease in the number of detected NPs; the loss in the number of detection events mainly originates from the decrease of the CG flow. The latter observation can be probably explained by the synergic effect of the lower aerosol production efficiency of the nebulizer, as well as the lower transport efficiency of the generated larger droplets by a lower carrier gas flow rate. 
a)

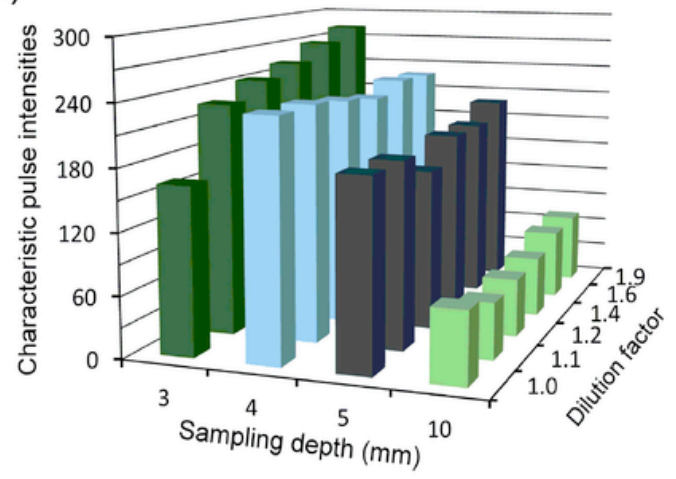

b)

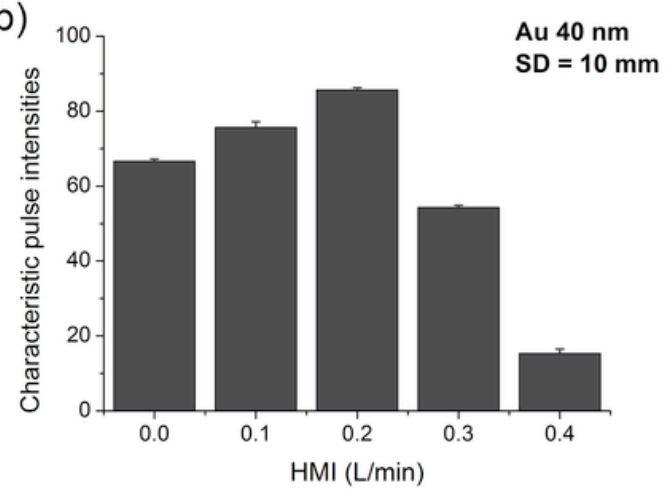

c)

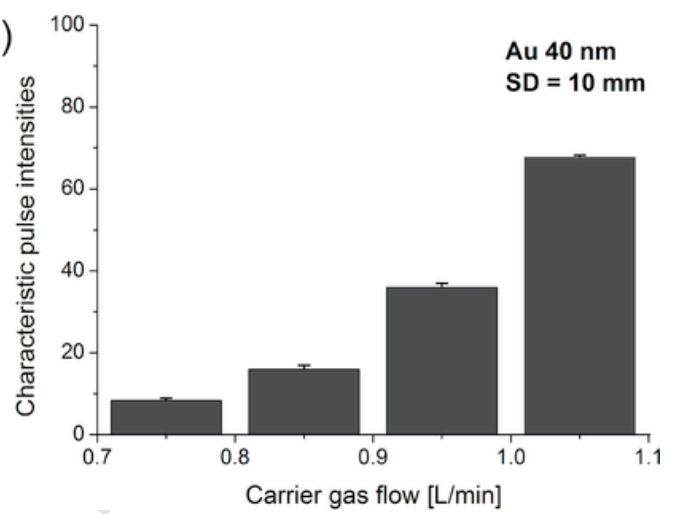

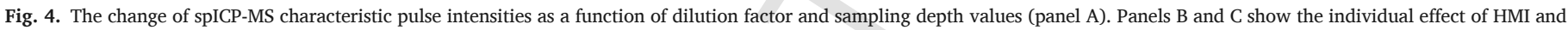

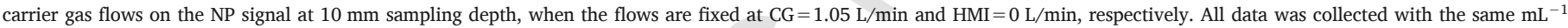
nanodispersion containing $40 \mathrm{~nm}$ Au NPs and keeping all other experimental conditions fixed. Error bars indicate the standard deviation calculated from three parallel measurements.

\section{Conclusions}

We carried out experiments to assess the separate and also the combined effect of the sampling depth and the aerosol gas flow rates on the performance of spICP-MS analysis on an Agilent 7700 instrument. It was found for both Ag and Au NPs that the NP signal can significantly be improved by the optimization of the plasma sampling depth - by decreasing it from the „robust” $8-10 \mathrm{~mm}$ value to $4 \mathrm{~mm}$, the signal can be nearly doubled. The optimal sampling depth value was the same for both NPs and the value agreed well with the optimum for solution-mode ICP-MS found for these two elements. This signal optimization was also found not to cause a deterioration of the signal repeatability, therefore a significant improvement in the size detection limit of nanoparticles could also be achieved. In the case of Au this improvement was $32 \%$, whereas for $\mathrm{Ag}$ it was $26 \%$. It was also observed that the histogram NP peaks not only shift upwards if the sampling depth is decreased, but they also become wider. However, the signal increase was found to be so strong that it overcompensated the peak widening, thus the peak separation in spICP-MS signal histograms actually increases (the ,,size resolution" increases). Thus, the optimization of the sampling depth was found to be a valuable tool in improving the performance of spICP-MS.
The effect of the aerosol dilution gas (called HMI option in Agilent nomenclature) was also studied as it was hoped to be useful for the on-line dilution of the aerosol generated from nano-dispersions, thereby making spICP-MS measurements more practical and faster. However, our experimental results prove that the HMI gas flow can only be used for aerosol dilution, if the total argon gas flow to the ICP plasma (the sum of the nebulizer/carrier gas flow and the HMI gas flow rate) is kept constant at its normal value. This means that the gas flow going to the pneumatic nebulizer needs to be proportionally lowered, thereby causing a significant worsening in the operation of the sample introduction system, which gives rise to a strong NP signal loss. The maximum volume dilution (approx. a factor of 2) achievable via the use of the HMI gas flow also does not seem to make the effort worth it. The aerosol gas dilution technique may be better used with nebulizers that either have separate gas flows for generating and carrying the aerosol or do not depend on a gas flow for aerosol generation.

\section{Appendix A. Supporting information}

Supplementary data associated with this article can be found in the online version at doi:10.1016/j.talanta.2017.05.051. 
a)
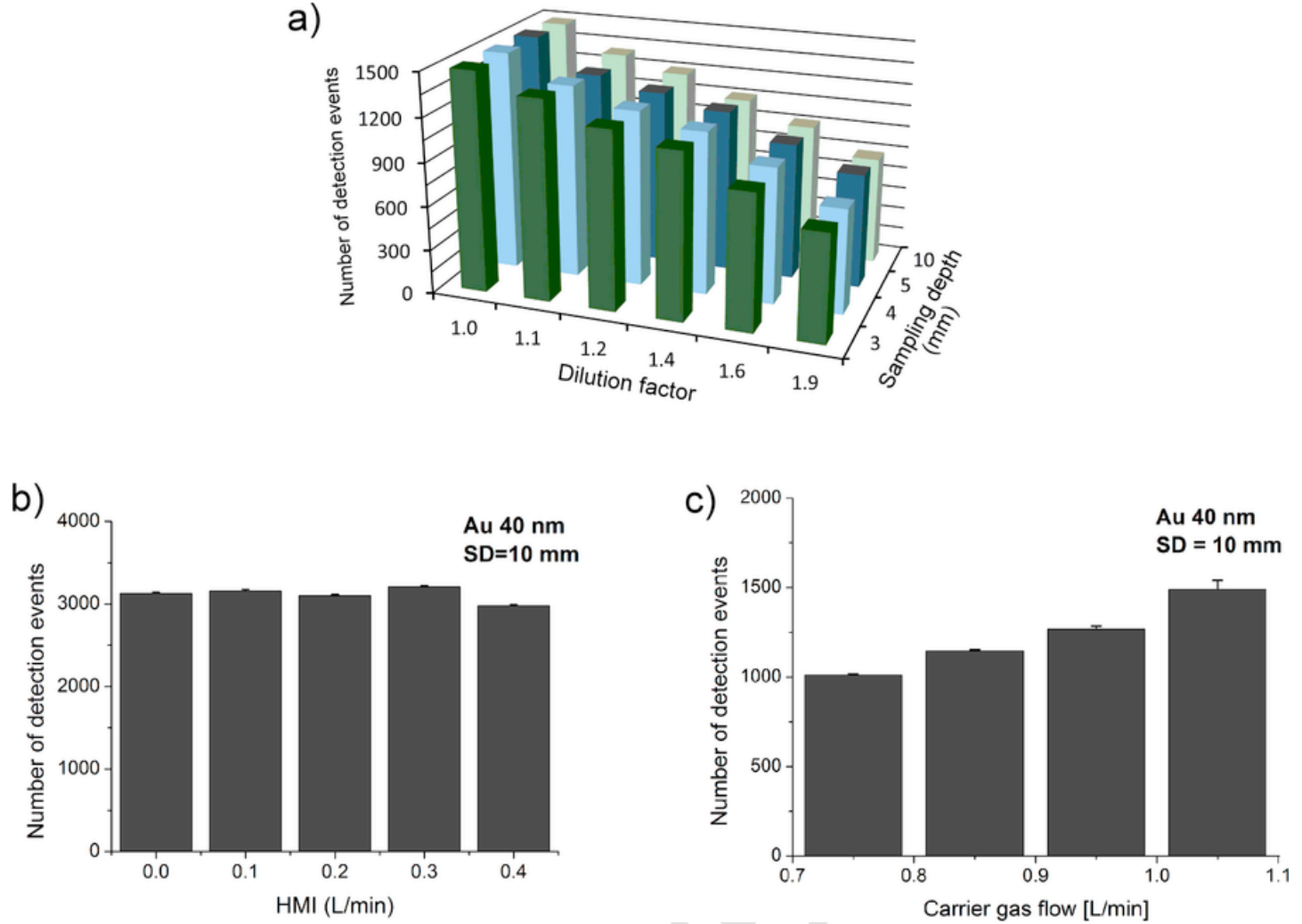

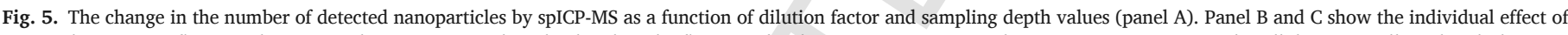

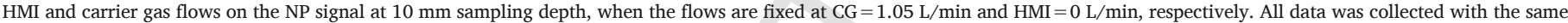
$\mathrm{mL}^{-1}$ nanodispersion containing $40 \mathrm{~nm}$ Au NPs and keeping all other experimental conditions fixed.

\section{References}

[1] F. Vanhaecke, P. Degryse (Eds.), Isotopic Analysis: Fundamentals and Applications Using ICP-MS, Wiley, 2012

[2] R. Thomas, Practical guide to ICP-MS, A tutorial for beginners 3rd ed, CRC Press, 2013.

[3] A. Limbeck, P. Galler, M. Bonta, G. Bauer, W. Nischkauer, F. Vanhaecke, Recent advances in quantitative LA-ICP-MS analysis: challenges and solutions in the life sciences and environmental chemistry, Anal. Bioanal. Chem. 407 (22) (2015) 6593-6617.

[4] J. Koch, D. Günther, Review of the state-of-the-art of laser ablation inductively coupled plasma mass spectrometry, Appl. Spectrosc. 65 (5) (2011) 155A-162A.

[5] D. Pröfrock, A. Prange, Inductively coupled plasma-mass spectrometry (ICP-MS) for quantitative analysis in environmental and life sciences: a review of challenges, solutions, and trends, Appl. Spectrosc. 66 (8) (2012) 843-868.

[6] G.K. Zoorob, J.W. McKiernan, J.A. Caruso, ICP-MS for elemental speciation studies, Microchim. Acta 128 (3) (1998) 145-168.

[7] C. Degueldre, P.-Y. Favarger, Colloid analysis by single particle inductively coupled plasma-mass spectroscopy: a feasibility study, Colloids Surf. A. 217 (1-3) (2003) 137-142.

[8] F. Laborda, E. Bolea, J. Jiménez-Lamana, Single Particle Inductively Coupled Plasma Mass Spectrometry: a Powerful Tool for Nanoanalysis, Anal. Chem. 86 (1-3) (2014) 2270-2278.

[9] M.D. Montaño, J.W. Olesik, A.G. Barber, K. Challis, J.F. Ranville, Single Particle ICP-MS: advances toward routine analysis of nanomaterials, Anal. Bioanal. Chem. 408 (19) (2016) 5053-5074.

[10] R.M. Galazzi, E. de Barros Santos, T. Caurin, G. de Souza Pessoa, I.O. Mazali, M.A.Z. Arruda, The importance of evaluating the real metal concentration in nanoparticles post-synthesis for their applications: a case-study using silver nanoparticles, Talanta 146 (2016) 795-800.

[11] I. Benesova, K. Dlabkova, F. Zelenak, T. Vakulovis, V. Kanicky, J. Preisler, Direct analysis of gold nanoparticles from dried droplets using substrate-assisted laser desorption single particle ICP-MS, Anal. Chem. 88 (5) (2016) 2576-2582.

[12] H.E. Pace, N.J. Rogers, C. Jarolimek, V.A. Coleman, E.P. Gray, C.P. Higgins, J.F. Ranville, Single Particle Inductively Coupled Plasma-Mass Spectrometry: a Performance Evaluation and Method Comparison in the Determination of Nanoparticle Size, Environ. Sci. Technol. 46 (22) (2012) 12272-12280.

[13] S. Lee, X. Bi, R.B. Reed, J.F. Ranville, P. Herckes, P. Westerhoff, Nanoparticle size detection limits by single particle ICP-MS for 40 elements, Environ. Sci. Technol. 48 (17) (2014) 10291-10300.

[14] I. Kálomista, A. Kéri, G. Galbács, On the applicability and performance of the single particle ICP-MS nano-dispersion characterization method in cases complicated by spectral interferences, J. Anal. At. Spectrom. 31 (5) (2016) 1112-1122.
[15] F. Vanhaecke, R. Dams, C. Vandecasteele, Zone model as an explanation for signal behaviour and non-spectral interferences in inductively coupled plasma mass spectrometry, J. Anal. At. Spectrom. 8 (3) (1992) 433-438.

[16] G. Horlick, H. Tan, M.A. Vaughan, C.A. Rose, The effect of plasma operating parameters on analyte signals in inductively coupled plasma-mass spectrometry, Spectrochim. Acta Part B 40 (10-12) (1985) 1555-1572.

[17] K. Niemax, Considerations about the detection efficiency in inductively coupled plasma mass spectrometry, Spectrochim. Acta B 76 (2012) 65-69.

[18] M.-A. Vaughan, G. Horlick, S.H. Tan, Effect of Operating Parameters on Analyte Signals in Inductively Coupled Plasma Mass Spectrometry, J. Anal. At. Spectrom. 2 (8) (1987) 765-772.

[19] M. Aghaei, H. Lindner, A. Bogaerts, Optimization of operating parameters for inductively coupled plasma mass spectrometry: a computational study, Spectrochim. Acta Part B 76 (2012) 56-64.

[20] G. Zhu, R.F. Browner, Investigation of Experimental Parameters with a Quadrupole ICP/MS, Appl. Spectrosc. 41 (3) (1987) 349-359.

[21] M. Aghaei, H. Lindner, A. Bogaerts, The effect of the sampling cone position and diameter on the gas flow dynamics in an ICP, J. Anal. At. Spectrom. 28 (9) (2013) 1485-1492.

[22] K.-S. Ho, W.-W. Lee, W.-T. Chan, Effects of ionization potential of an element and boiling point of the corresponding oxide on the sensitivity of ICP-MS, J. Anal. At. Spectrom. 30 (10) (2015) 2066-2073.

[23] S. Wilbur, C. Jones, Simple, Reliable Analysis of High Matrix Samples According to US EPA Method 6020A using the Agilent 7700x ICP-MS, Application Note No. 5990-5514EN, Agilent, 2010.

[24] B.H. Kim, M.J. Hackett, J. Park, T. Hyeon, Synthesis, Characterization, and Application of Ultrasmall Nanoparticles, Chem. Mater. 26 (1) (2014) 59-71.

[25] K. Zarschler, L. Rocks, N. Licciardello, L. Boselli, E. Polo, K.P. Garcia, L. De Cola, S. Holger, K.A. Dawson, Ultrasmall inorganic nanoparticles: state-of-the-art and perspectives for biomedical applications, Nanomed.: Nanotechnol., Biol., Med. 12 (6) (2016) 1663-1701.

[26] O.V. Kharissova, B.I. Kharisov, V.M. Jiménez-Pérez, B. Muñoz Flores, U.O. Méndez, Ultrasmall particles and nanocomposites: state of the art, RSC Adv. 3 (2013) 22648-22682.

[27] S. Sannac, S. Tadjiki, E. Moldenhauer, Single particle analysis using the Agilent 7700x ICP-MS, Application Note No. 5991-2929EN, Agilent, 2013.

[28] J.W. Olesik, P.J. Gray, Considerations for measurement of individual nanoparticles or microparticles by ICP-MS: determination of the number of particles and the analyte mass in each particle, J. Anal. At. Spectrom. 27 (7) (2012) 1143-1155.

[29] W.-W. Lee, W.-T. Chan, Calibration of single-particle inductively coupled plasma-mass spectrometry (SP-ICP-MS), J. Anal. At. Spectrom. 30 (6) (2015) 1245-1254.

[30] D. Kutscher, J.D. Wills, S. McSheeny-Ducos, Analysis of high matrix samples using argon gas dilution with the Thermo Scientific ICAP Q ICP-MS, Technical Note No. 43202, Thermo Scientific, 2014. 
[31] New All Matrix Solution (AMS) System for NexION ICP-MS, Product Note No. 009539_01, Perkin Elmer, 2011.

[32] S.E. Maestre, J.L. Todoli, J.M. Mermet, Evaluation of several pneumatic micronebulizers with different designs for use in ICP-AES and ICP-MS. Future directions for further improvement, Anal. Bioanal. Chem. 379 (5) (2004) 888-899.

[33] J. Sneddon (Ed.), Sample Introduction in Atomic Spectroscopy, Elsevier, 1990.

[34] L. Flamigni, J. Koch, D. Günther, Spectrochim. Acta B 76 (2012) 70-76.

[35] G.C.-Y. Chan, Z. Zhu, G.M. Hieftje, Development and evaluation of discrete sample introdution system for nanoparticle analysis by ICP-MS, Spectrochim. Acta B 76 (2012) 77-86.

[36] K.-S. Ho, K.-O. Lui, K.-H. Lee, W.-T. Chan, Considerations of particle vaporization and analyte diffusion in single-particle inductively coupled plasma-mass spectrometry, Spectrochim. Acta Part B 89 (2013) 30-39.

[37] G. Cornelis, M. Hassellöv, A signal deconvolution method to discriminate smaller nanoparticles in single particle ICP-MS, J. Anal. At. Spectrom. 29 (1) (2014) 134-144.

[38] F. Laborda, M.S. Jiménez, E. Bolea, J. Castillo, An approach to the natural and engineered nanoparticles analysis in the environment by inductively coupled plasma mass spectrometry, Int. J. Mass Spectrom. 307 (1-3) (2011) 99-104.

[39] J. Liu, K.E. Murphy, R.I. MacCuspie, M.R. Winchester, Capabilities of single particle inductively coupled plasma mass spectrometry for the size measurement of nanoparticles: a case study on gold nanoparticles, Anal. Chem. 86 (7) (2014) 3405-3414.

[40] R.B. Reed, C.P. Higgins, P. Westerhoff, S. Tadjikid, J.F. Ranville, Overcoming challenges in analysis of polydisperse metal-containing nanoparticles by single particle inductively coupled plasma mass spectrometry, J. Anal. At. Spectrom. 27 (7) (2012) 1093-1100.
[41] J.F.J. Todd, Recommendations for nomenclature and symbolism for mass spectroscopy (including an appendix of terms used in vacuum technology), Pure Appl. Chem. 63 (10) (1991) 1541-1566.

[42] F. Laborda, J. Jimenez-Lamana, E. Bolea, J.R. Castillo, Selective identification, characterization and determination of dissolved silver(I) and silver nanoparticles based on single particle detection by inductively coupled plasma mass spectrometry, J. Anal. At. Spectrom. 26 (7) (2011) 1362-1371.

[43] M. Aghaei, H. Lindner, A. Bogaerts, Ion clouds in the inductively coupled plasma torch: a closer look through computations, Anal. Chem. 88 (16) (2016) 8005-8018.

[44] R.L. Spencer, J. Krogel, J. Palmer, A. Payne, A. Sampson, W. Somers, C.N. Woods, Modeling the gas upstream and in the sampling nozzle of the inductively coupled plasma mass spectrometer via the Direct Simulation Monte Carlo algorithm, Spectrochim. Acta Part B 64 (3) (2009) 215-221.

[45] R.L. Spencer, N. Taylor, P.B. Farnsworth, Comparison of calculated and experimental sampling cone of an inductively coupled plasma mass spectrometer, Spectrochim. Acta Part B 64 (9) (2009) 921-924.

[46] M. Aghaei, L. Flamigni, H. Lindner, D. Günther, A. Bogaerts, Occurrence of gas flow rotational motion inside the ICP torch: a computational and experimental study, J. Anal. At. Spectrom. 29 (2) (2014) 249-261. 